\title{
Renal Clearance Normalized by BMI
}

National Cancer Institute

\section{Source}

National Cancer Institute. Renal Clearance Normalized by BMI. NCI Thesaurus. Code

C105458.

The rate at which a substance is removed from the blood via the kidneys, divided by the body mass index. 